\title{
A SURVEY OF SPIKING NEURAL NETWORKS AND SUPPORT VECTOR MACHINE PERFORMANCE BYUSINGGPU'S
}

\author{
Israel Tabarez-Paz ${ }^{1}$, Neil Hernández-Gress ${ }^{2}$ and Miguel González Mendoza ${ }^{2}$. \\ ${ }^{1}$ Universidad Autónomadel Estado de México \\ Blvd. Universitario s/n, Predio San Javier Atizapán de Zaragoza, \\ México \{itabarezp\}@uaemex.mx \\ http://www. uaem.mx/cuyuaps/vallemexico.html \\ ${ }^{2}$ Tecnológico de Monterrey, Campus Estado de México, \\ CarreteraLago de Guadalupe km 3.5 Atizapán de Zaragoza Col. Margarita Maza de \\ Juarez, Atizapán de Zaragora, México \\ \{ngress, mgonza\}@itesm.mx $\square$ http://www.itesm.edu
}

\begin{abstract}
In this paper we study the performance of Spiking Neural Networks (SNN)and Support Vector Machine (SVM) by using a GPU, model GeForce 6400M. Respect to applications of SNN, the methodology may be used for clustering, classification of databases, odor, speech and image recognition..In case of methodology SVM, is typically applied for clustering, regression and progression. According to particular characteristics of these methodologies, theycan be parallelizedin several grades. However, level of parallelism is limited to architecture of hardware. So, is very sure to get better results using other hardware with more computational resources. The different approaches are evaluated by the training speed and performance. On the other hand, some authors have coded algorithms SVM light, but nobody has programming QP SVM in a GPU. Algorithms were coded by authors in the hardware, like Nvidia card, FPGA or sequential circuits that depends on methodology used, to compare learning timewith between GPU and CPU. Also, in the survey we introduce a brief description of the types of ANN and its techniques of execution to be related with results of researching.
\end{abstract}

\section{KEYWORDS}

GPU,Spiking Neural Networks, Support Vector Machines, pattern recognition.

\section{INTRODUCTION}

This paper presents a surveyof Spiking Neural Networks (SNN) and Support Vector Machine (SVM) by using a GPU, model GeForce 6400M. In case of SNN, this methodology started to be developed by Hodgkin - Huxleysince 1930whose model has four differential equations with partial non linear derivatives, and depends on the space and time. This describes propagation and generation of potential of a big axon of squid in order to explain the main properties. SNN's is the model most similar to the neurons of mammals[27].SNN can be applied to the same problems that depend on behavior of time of parameters because of its singular characteristic of coding in the time[13]. In case of SVM, in decade of 1990's was started the development[34], even this methodology had been invented since 1979 [31] by Vapnik, to solve more complex problems,linearlly separable or non - separable. SVM uses strategies of optimization to get the global solution however, this consume big quantities of computational resources.

DOI: $10.5121 /$ ijsc. 2013.4301 
Some trends of topics are about ANN is Parallel Programming to solve problems such as clustering (Herrero-Lopez[8]), pattern recognition (Olaf [27]), regression (Carpenter[19]), building of ANN in a specific hardware, such as FPGAs (Papadonikolakis[7]). The perceptron is the first training unit in which a training algorithm for linearly separable problems, that consist of a single neuron. Mathematically, itis represented by a straight-line equation[35]. However, non linear problems can't be solved with this methodology.

In 2003 was developed the methodology SNN also called Spike Response Model (SRM) (Bohte[28], Olaf[27]). At the same time,Izhikevich[29] developed a reduced model of Hodgkin Huxley model that consist on two differential equations that explain behavior of mammal neurons. The main diference between SRM and Izhikevich's model are the differential equations.

Respect to parallel programming[37], calculations are carried out simultaneously, operating on the principle that large problems can often be divided into smaller ones, that are solved concurrently. There are several different forms of parallel computing: bit-level, instruction level, data, and task parallelism. This manuscript is focused on instruction level and task. In the figure 1 , we can see principle of parallel computing.

Problems of parallel programming can be solved in OpenGL, Cg, C, C++ and Fortran. Finally, they are developed for CUDA language.

This paper is distributed as follows: in section 2 the state of the art ofANN is presented, in section 3 the performance of ANN in parallel programming is included, finally in section 4 are the conclusions.

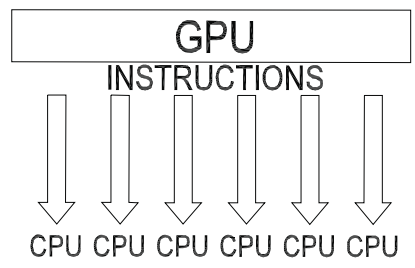

Figure 1.Parallel programming.

\section{RELATING WORKS ON THE USE OF PARALlel PROGRAMMING IN Artificial NeUral Networks}

Recently works about SNN are shown in the next figure.
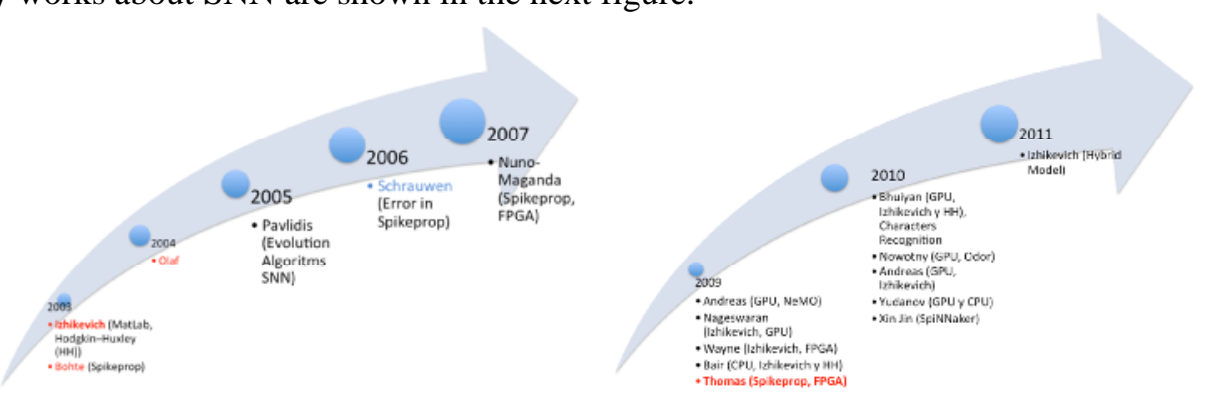

Figure 2.State of the Art of SNN. 
Stewart and Bair 0, he Applies Runge - Kutta's method for Izhikevich and Hodgkin Huxle's models. As a result, second method is more efficient

Thomas and Luk[18], the author proposes a Simulation with maximum 1024 neurons in a FPGA the Izhikevich's model.

Nageswaran [17], he presents a compilation of theIzhikevich's models.

Papadonikolakis[15], he has focused on improving the speed of learning and efficiency of SVMs using several methods. Also, he compares these parameters between a GPU and a FPGA programming Gilbert' algorithm.

Yudanov[13], implements a hybrid method with numeric integration of Parker Sochacki (PS) with adaptative order. This is validated at the moment in the comparision made between GPU and CPU in their characteristics.

Bhuiyan[11], compares the models of SNN such as Izhikevich and Hodgkin Huxley, these models applied to recognition of characters.

Izhikevich[9], designs a hybrid model for SNN in order to combine continuous and discontinuous numeric methods.

Scanzio[12], He compares the speed of processing in CUDA of algorithms feedfordward and backpropagation.

Prabhu[20], He applies GPU for pattern classifier in images. He focuses on the degree of parallelism of a problem. He uses maximum size of image of $256 \mathrm{MB}$, and in a video memory GPU of $768 \mathrm{MB}$. As a result, author compares Dual - Core AMD processor with a Geforce 6150 GPU, and when the number of patterns increase, the CPU is linearly slower than GPU, But when the network size increase the curve isn't linear.

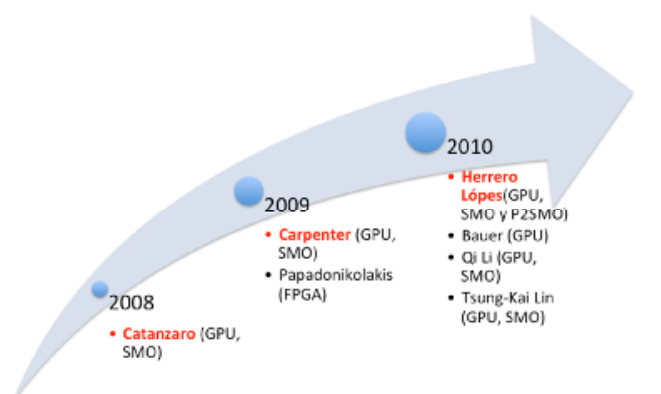

Figure 3. State of the Art of SVM

MarkosPapadonikolakis[7], he proposes to implement a FPGA for accelerate SVM for classifying.

Catanzaro [21], the author proposes the SVM using algorithm of Sequential Minimal Optimization (SMO), also he compares time of learning and precision of classification between GPU and libraries of SVM of MatLab

Austin Carpenter [19], he applies cuSVM for NVIDIA with a modified version of SMO.

Sergio HerreroLópez[8], he made classification using SVM in a GPU. He continues the work of Catanzaro.

\section{PARALLEL PROGRAMMING FOR SVM AND SNN}

According to Thomas [18], Artificial Neural Networks (ANN) can be parallelized with a GPU, FPGAspecificcard [14],SVM's are wellimplementedin a GPUbecause of optimizing methodrequires of solving repetitively operations of matrixes. However, for SNN methodology is better to use FPGA or sequential circuits, although it can be simulated in GPU. The reason is that in a GPU thelearning time is calculated with a non linear and exponential equation, which algebraic 
order depends directly of number of neurons in the input. This equation is solved with mathematical approximations, so if the number of neurons in an input layer is increasing, the computational complexity too. However, to design SNN architecture in the circuit mentioned, the time does not need a mathematical solution to find the threshold, this is only detected with a simplex circuit.

\subsection{Considerations to start with a GPU}

This section is focused in program CUDA in a GPU. Respect to state of the art, configuration of a GPU depends on limitations of the hardware, so authorstake into account other investigations as reference to improve the algorithms.

An important problemisto configure the hardware selected. In this case, SNN can be programmed with kernel in three dimensions. The simplest configuration in one or two dimensions as perceptron method.

Starting to work with a GPU, we have to know how many threads, blocks and grids we need to use. In case of SNN each block can represent only one neuron. Sometimes computational resources are not enough. Other problem ifthemethod is recursive and weightsmust be frequently adjusted because of local memory couldnotbe enough.

\subsection{Levelof parallelization}

In case of perceptron, allneuronsperlayer can be calculated at the same time because of thesimple form of its activation function. On the other hand, each layer must be calculated sequentially because of input of hidden layer depends on output of previous layer. However, this activity seems like FIFO principle (First in - First out).

SVM depends of quantity of instances, because of this methodology spend a thread per data of the matrix, but the size of the matrix is the quantity of dataspow two or three. This imply reduce parallelization of the algorithm when data are approximately more than 16 instances.

SNN an implement algorithmof a mathematical or sequential method to calculate the value of threshold in amplitude and time is needed. This impliesthatmany values of time are needed, as well as, maybe hundreds or thousands per row in a block. So, a neuron is represented as a grid in tree dimensions, where each row can represent a previous neuron to be added for one output of the following neuron. This method requiresgood memory resources.

\subsection{Algorithms for parallelization}

Parallelization of ANN consists in two phases: first learning phase, and second execution phase. Parallelization for learning phase in case of SVM is in parallelization of matrixes operations, what is well defined in webpage of CUDA[37], although this depends on optimization method applied what is focused on solving quadratic problem[38]. In case of SNN, the mathematical calculus in phase of learning is more sequential because of its approximation method.But in both SVM and SNN all weight of hidden layers neurons can be parallelized and calculated at the same time.

The second phase refers about to evaluations of weights calculated according to inputs. The sections $\mathrm{A}, \mathrm{B}$ and $\mathrm{C}$ show a way of parallelizing of this phase. 
Preceptron.Graphically, the following components of the model represent the actual activity of a neuron. All inputs are summed altogether and modified by the weights. This activity is referred as a linear combination. Finally, an activation function controls the amplitude of the output. This process is described in the figure 2. Each thread represents a layer. In the GPU, parallelism for a neuron of perceptron is focused on mathematical operations[37].

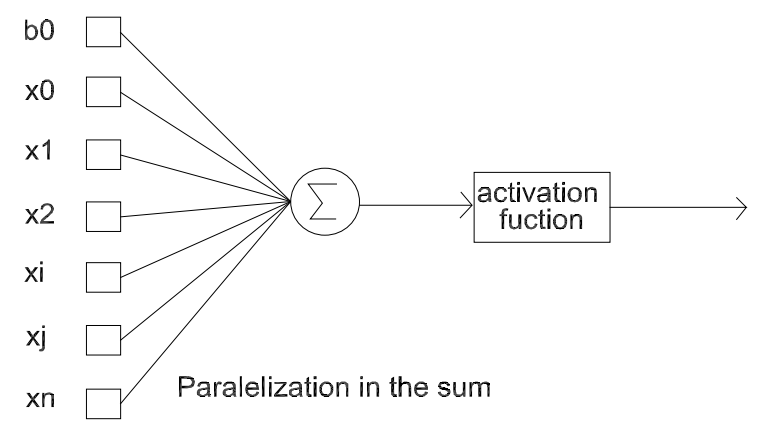

Figure 2.Simple perceptron.

Backpropagation.In this case has the samealgorithm in weight thatSNN. The weight needs to be adjusted per iteration. Parallelization in two dimensionsis like the figure 3.

Support Vector Machines (SVM).In the figure 3, the parallelization of SVM could be observed, in this case each thread represents a layer with $\mathrm{n}$ or $\mathrm{k}$ number of rows, however the mathematics calculus are parallelized into of each neuron of hidden layer in other kernel, what is solved as a optimization problem. The dimension of the Hessian matrix isequal to numberof input parameters. So, the multiplication of matrix is another operation to parallelize that can be solved in a separated kernel. In the figure 3 , the y0 neuron gets at the same time all values multiplied per its respective weight, the in other kernel in CUDA the mathematical operations are parallelized. At the same time the other neurons of hidden layer computing its respective output. However, the next layer cannot calculate its output without the previous layer has done.

Figure 3. Parallelization of Support Vector Machine (SVM), 2D Array

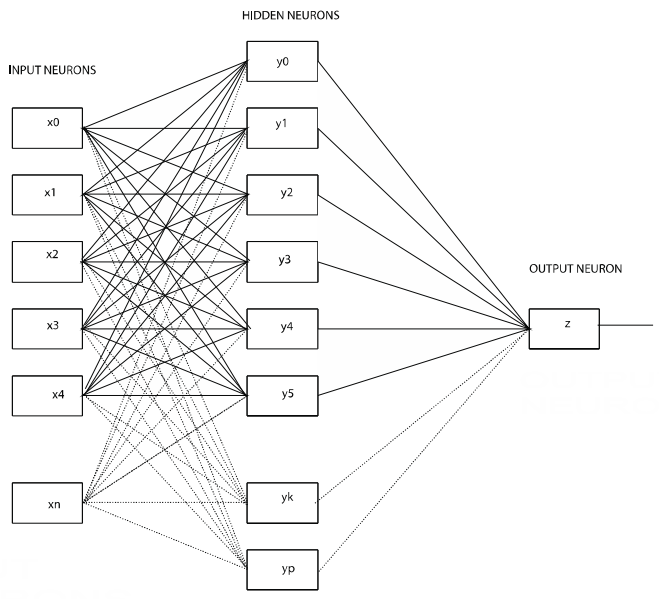


Spiking Neural Networks (SNN).Thearrange of figure 4 represents a configuration of the GPU device in three dimensions. This is a solution for parallelizing SNNalgorithm. The cube showedin this figure is only a neuron of a hidden or output layer.There are as many cubes as neurons are required. Each cube is divided in blocks, what depend on the length of time in the input[27]. All neurons per layer can be calculated in parallel, but a disadvantage is thatthis procedure requires many resources of memory. .

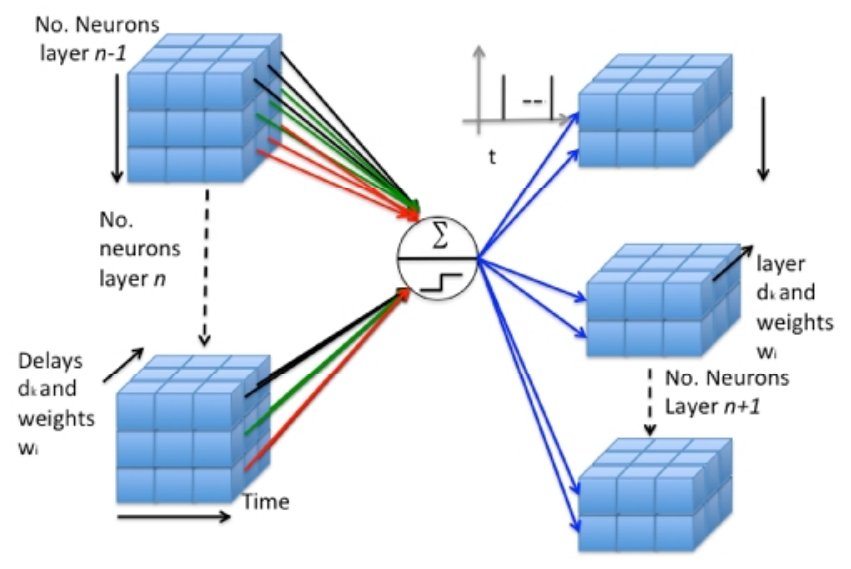

Figure 4.Parallelization in 3 dimensions of Spiking Neural Network.

SNN has significant characteristics that must be considerated. The synapses of the biological neuron are modeled as weights. Let's remember that the synapse of the biological neuron is which interconnects the neural network and gives the strength of the connection. For an artificial neuron, the weight is a number, and represents the synapse. A negative weight reflects an inhibitory connection, while positive values designate excitatory connections.Inherent parallelism of commodity graphic hardware is used to accelerate the computationofANN.According to Nikola[33], taxonomy of parallelization approaches for neurosimulations is represented in the figure 5 .

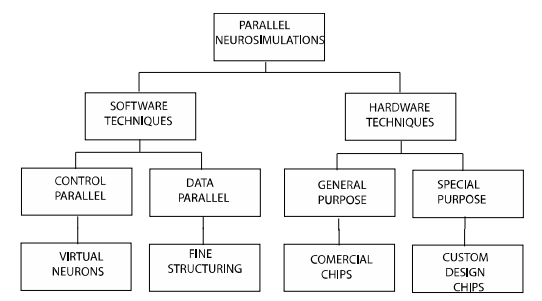

Figure 5. Taxonomy of parallelization approaches for neurosimulations.

Sridhar [1] says that the main advantage of GPU over CPU is high computational parallelism and efficiency with a relatively low cost.However, it is difficult to design an algorithm. Also, the author says that although exist Integrated Circuits (IC) for high parallelism, it is very difficult to translate this parallelism in an efficient software. On the other hand, human brain can be trained to solve complex problems, such as thermal modeling of specific IC layouts.

Prabhu [20], compares efficiency of the human brain with enormous computational powerand parallel environs of GPU's, so we understand that GPU has some limitations. According to him, the role played by Graphical Processing Unit (GPU) is approaching to Artificial Neural Networks to the nature of human brain. Also, GPU's have been used for rendering high quality images in 
real time, virtual reality simulations and games. Modern GPU's can perform highly intensive parallel tasks.

\section{Conclusions}

In this paper weconcludethatparallelisminANNincreasespeedof learning time. However, is very difficult to design this sort of algorithms. On the other hand, we can parallelize by hardware (FPGA) of software (GPU). Tendency is study to know which algorithm is the most efficient and faster, because of their mathematical characteristics and their architecture. So, is better to solve a problem with large database using SVM and SNN than traditional ANN.

The importance to compare the efficiency between these algorithms is to know the error in the results and which is faster for learning according to quantity of instances and parameters per instance. So, with this information is possible to know what applications are the most appropriates for each application.

As a future work, there are some aspects, as parallelizing SVM or SNN in a GPU and SNN in a FPGA, then compare learning time. However, also is necessary to propone an important application to solve real problems.

\section{REFERENCES}

[1] Sridhar, A.; Vincenzi, A.; Ruggiero, M.; Atienza, D.; , "Neural Network-Based Thermal Simulation of Integrated Circuits on GPUs," Computer-Aided Design of Integrated Circuits and Systems, IEEE Transactions on , vol.31, no.1, pp.23-36, Jan. 2012

[2] Ahmadi, Arash; Soleimani, Hamid; , "A GPU based simulation of multilayer spiking neural networks," Electrical Engineering (ICEE), 2011 19th Iranian Conference on , vol., no., pp.1-5, 17-19 May 2011

[3] Lowe, E.W.; Woetzel, N.; Meiler, J.; , "Poster: GPU-accelerated artificial neural network for QSAR modeling," Computational Advances in Bio and Medical Sciences (ICCABS), 2011 IEEE 1st International Conference on , vol., no., pp.254, 3-5 Feb. 2011

[4] B. Kirk, David; W. Hwu, Wen-mai "Programming Massively Parallel Processors" Ed. Morgan Kaufmann, 2010.

[5] Qi Li; Salman, R.; Kecman, V.; , "An intelligent system for accelerating parallel SVM classification problems on large datasets using GPU," Intelligent Systems Design and Applications (ISDA), 2010 10th International Conference on , vol., no., pp.1131-1135, Nov. 29 2010-Dec. 12010

[6] Tsung-Kai Lin; Shao-Yi Chien; , "Support Vector Machines on GPU with Sparse Matrix Format," Machine Learning and Applications (ICMLA), 2010 Ninth International Conference on , vol., no., pp.313-318, 12-14 Dec. 2010

[7] Papadonikolakis, M.; Bouganis, C.: "A novel FPGA-based SVM classifier," Field-Programmable Technology (FPT), 2010 International Conference on , vol., no., pp.283-286, 8-10 Dec. 2010

[8] Sergio Herrero-Lopez, John R. Williams, Abel Sanchez,: Parallel Multiclass Classification using SVMs on GPUs, November 2010.

[9] Izhikevich, E.M.: "Hybrid spiking models", vol. 368, issue 1930, pp. 5061-5070, Nov 2010

[10] VenkittaramanVivek, PallipuramKrishnamani: ACCELERATION OF SPIKING NEURAL NETWORKS ON SINGLE- GPU AND MULTI-GPU SYSTEMS, ProQuest document ID: 204037751, Publication Number: AAT 147555, May 2010.

[11] Bhuiyan, M.A.; Pallipuram, V.K.; Smith, M.C.: "Acceleration of spiking neural networks in emerging multi-core and GPU architectures," Parallel \& Distributed Processing, Workshops and Phd Forum (IPDPSW), 2010 IEEE International Symposium on , vol., no., pp.1-8, 19-23 April 2010

[12] Scanzio, S.; Cumani, S.; Gemello, R.; Mana, F.; Laface, P.; , "Parallel implementation of artificial neural network training," Acoustics Speech and Signal Processing (ICASSP), 2010 IEEE International Conference on , vol., no., pp.4902-4905, 14-19 March 2010. 
[13] Yudanov, D.; Shaaban, M.; Melton, R.; Reznik, L.; GPU-Based Simulation of Spiking Neural Networks with Real-Time Performance \& High Accuracy, Feb 2010.

[14] XIN JIN: Parallel Simulation of Neural Networks on Spinnaker Universal Neuromorphic Hardware, University of Manchester, 2010

[15] Papadonikolakis, M.; Bouganis, C.-S.; Constantinides, G.: "Performance comparison of GPU and FPGA architectures for the SVM training problem," Field-Programmable Technology, 2009. FPT 2009. International Conferenceon, vol., no., pp.388-391, 9-11 Dec. 2009

[16] Fidjeland, A.K.; Roesch, E.B.; Shanahan, M.P.; Luk, W.; , "NeMo: A Platform for Neural Modelling of Spiking Neurons Using GPUs," Application-specific Systems, Architectures and Processors, 2009. ASAP 2009. 20th IEEE International Conference on , vol., no., pp.137-144, 7-9 July 2009

[17] Nageswaran, J.M., Dutt, N.: Krichmar, J.L.; Nicolau, A.; Veidenbaum, A.; , "Efficient simulation of large-scale Spiking Neural Networks using CUDA graphics processors," Neural Networks, 2009. IJCNN 2009. International JointConferenceon, vol., no., pp.2145-2152, 14-19 June 2009

[18] Thomas, D.B.;Luk, W.: "FPGA Accelerated Simulation of Biologically Plausible Spiking Neural Networks," Field Programmable Custom Computing Machines, 2009. FCCM '09. 17th IEEE Symposium on , vol., no., pp.45-52, 5-7 April 2009

[19] Carpenter, A.; "CUSVM: A CUDA IMPLEMENTATION OF SUPPORT VECTOR CLASSIFICATION AND REGRESSION", Jan. 2009.Stewart R.D., Bair W.: "Spiking neural network simulation: numerical integration with the Parker-Sochacki method", Jan. 2009

[20] Prabhu, R.D.; "SOMGPU: An unsupervised pattern classifier on Graphical Processing Unit," Evolutionary Computation, 2008. CEC 2008.(IEEE World Congress on Computational Intelligence). IEEE Congress on, vol., no., pp.1011-1018, 1-6 June 2008

[21] Bryan Catanzaro, Narayanan Sundaram, KurtKeutzer: Fast Support Vector Machine Training and Classification on Graphics Processors, International Conference on Machine Learning, Helsinki, Finland, 2008.

[22] Martínez Z. M., Díaz P. F. J., Díez H. J. F, Antón R. M.: Fuzzy ART Neural Network Parallel Computing on the GPU, Springer (2007).

[23] Philipp S., Grübl A., Meier K., Schemmel J.: Interconnecting VLSI Spiking Neural Networks Using Isochronous Connections, Springer (2007)

[24] L. Zhongwen, L. Hongzhi and W. Xincai: Artificial Neural Network Computation on Graphic Process Unit (2005).

[25] N.G. Pavlidis, D.K. Tasoulis, V.P. Plagianakos, G. Nikiforidis, M.N. Vrahatis: Spiking Neural Network Training Using Evolutionary Algorithms, IEEE International Joint Conference on, pp: 2190 -2194, vol. 4 , december 2005.

[26] SeijasFossi C., Caralli D’ Ambrosio A.: Uso de lasmáquinas de soportepara la estimación del potencial de acción cellular, Revista de Ingeniería UC. Vol. 11, Nº 1, 56 - 61 (2004).

[27] Olaf Booij,: Temporal Pattern Classification using Spiking Neural Networks, Intelligent Sensory Information Systems Informatics Institute Faculty of Science Universiteit van Amsterdam (2004).

[28] Sander M. B.: Spiking Neural Networks, Universiteit Leiden (2003).

[29] Izhikevich, E.M.: "Simple model of spiking neurons," Neural Networks, IEEE Transactions on , vol.14, no.6, pp. 1569- 1572, Nov. 2003

[30] Sander M. Bohte, Joost N. Kok, Han La Poutre: Error-backpropagation in temporally encoded networks of spiking neurons, Neurocomputing 48, pp: $17-37$, (2002).

[31] Platt C. J.: Sequiential Minimal Optimization: A fast Algorithm for Tarining Support Vector Machines (1998).

[32] Osuna E., Freund R., Girosi F.: An Improved Training Algorithm for Support Vector Machines, In Proc. of IEEE NNSP'97.

[33] Nikola B. Serbediija: Simulating Artificial Neural Networks on Parallel Architectures (1996).

[34] Vapnik V., Cortes C., Support Vector Networks (1995).

[35] FausetLaurene, Fundamentals of Neural Networks, ARCHITECTURE, ALGORITHMS, AND APPLICATIONS, Prentice Halls, 1994.

[36] A. L. Hodgkin and A. F. Huxley: A quantitative description of membrane current and its application to conduction and excitation in nerve, J. Physiol. (1952).

[37] http://www.nvidia.com

[38] http://www.svms.org 


\section{Authors}

M en C Israel Tabarez Paz: He works for Universidad Autónomadel Estado de Méxicoas a researcher. He is focus on Artificial Intelligence, Artificial Neural Networks. Also he is a PhD. Student in Computing M en C Israel Tabarez Paz: He works for Universidad Autónomadel Estado de Méxicoas a researcher. He is focus on Artificial Intelligence, Artificial Neural Networks. Also he is a PhD. Student in Computing Science in InstitutoTecnológico y de EstudiosSuperiores de Monterrey Campus Estado de México (ITESM).

PhD. Neil Hernández Gress:He works for de InstitutoTecnológico y de EstudiosSuperiores de Monterrey Campus Estado de México (ITESM)as a Director of Research Technological Development and Postgrade in ITESM. He is focus on Artificial Intelligence, Artificial Neural Networks.

$\mathrm{PhD}$. Miguel González Mendoza.Head of the P Program in Computer Sciences and Engineering in ITESM. Secretary in Mexican Society on Artificial Intelligence 